\section{Avaliação dos serviços de urgência e emergência da rede hospitalar de referência no Nordeste brasileiro}

\author{
Evaluation of urgent and emergency services in the \\ hospital referral system in Northeast Brazil
}

\begin{abstract}
${ }^{1}$ Centro de Pesquisas Aggeu Magalhães, Fundação Oswaldo Cruz, Recife, Brasil. 2 Instituto de Medicina Integral Prof. Fernando Figueira, Recife, Brasil.

Correspondência L. S. Dubeux Centro de Pesquisas Aggeu Magalhães, Fundação Oswaldo Cruz.

Rua Dom João Costa 274 Recife, PE 52030-220, Brasil. lucianadubeux@yahoo.com.br
\end{abstract}

Abstract

This article evaluates the degree of implementation of ten urgent and emergency hospital services comprising the macro and micro-regional referral system in Pernambuco State, Northeast Brazil. The study analyzes criteria related to structure (physical and organizational, and material and human resources) and process (routine and referral/counter-referral activities), classifying the hospitals as satisfactory, acceptable, and deficient. The majority of the hospitals were classified as deficient, including all the micro-regional units and one macro-regional referral unit. Macro-regional units show better performance in the work process, while implementation of the structural dimension is better in the micro-regional hospitals. The results highlight the priority of upgrading these hospitals by strengthening decentralized human resources and technology policies, oriented towards the improvement of work processes in keeping with the State's regional contexts.

Hospital Emergency Service; Health Evaluation; Regional Health Planning
Luciana Santos Dubeux 1,2

Eduardo Freese 1

Yluska Almeida Coelho dos Reis 1

\section{Introdução}

A partir da década 1980, com a redemocratização do país, refletida na promulgação da Constituição Federal de 1988, o Estado Federativo Brasileiro foi recuperando as bases próprias de autonomia política para todas as esferas de governo, repercutindo na descentralização de políticas setoriais e promovendo novas conformações entre as instâncias governamentais. No cenário de Reforma das Políticas de Proteção Social, a descentralização da assistência à saúde acompanha a descentralização das políticas sociais 1 , havendo a desconcentração, da União para os municípios, de recursos financeiros e da prerrogativa de administração da atenção ambulatorial e hospitalar, em um processo que secundarizou o papel da gestão estadual 2 .

A forte característica municipalista conduziu à atomização de sistemas municipais de saúde, na compreensão de Lucchese 3 , ou municipalização autárquica na concepção de Mendes 4. Como efeito, observa-se a fragilidade da gestão e prestação de serviços nos sistemas regionais e estaduais de saúde, especialmente no que se refere à organização das ações de média e alta complexidade assistencial. Diante deste contexto, desencadeou-se no país a publicação de aparatos normativos e legais, com ênfase para o Pacto pela Saúde ${ }^{5}$, que na definição da atribuição de políticas públicas para as esferas locais, 
direcionou esforços para o fortalecimento da regionalização.

Normativamente, tais instrumentos subsidiaram o processo de resignificação do papel da esfera estadual no planejamento, gestão e avaliação das relações intermunicipais e dos espaços regionais de saúde, ampliando a produção e a produtividade das ações e serviços no Sistema Único de Saúde (SUS). A despeito disto, verificase a persistência de necessidades estruturais nas microrregiões. Estes são espaços prósperos que acumulam alguns êxitos visíveis, podendo permitir a consecução de mudanças significativas no SUS 6, devido à aproximação com as necessidades e demandas micro e loco-regionais.

Neste cenário, considerando os dinâmicos processos de transição demográfica e epidemiológica, com o aumento da expectativa de vida e da prevalência de doenças crônicas e degenerativas, da explosão da violência urbana nos municípios de médio e grande porte e o incremento acentuado de acidentes em rodovias estaduais e federais; a assistência hospitalar regionalizada configura-se como primeira referência para causas externas, agravos agudos e eventos decorrentes de doenças crônicas.

Diante da prioridade desta situação, as iniciativas públicas federais para estruturação de Sistemas Regionalizados de Urgência e Emergência são marcadas pela implantação de programas para qualificação dos aspectos estruturais e dos processos de trabalho, destacando-se o Sistema Estadual de Urgência e Emergência em 2002 7, o Projeto de Investimentos para a Qualificação do Sistema Único de Saúde (QualiSUS) 8, instituído em 2004, a organização de Redes Loco-regionais de Atenção Integral às Urgências regulamentada em 2008 9, entre outros. Em conjunto, tais estratégias buscam a incorporação de avanços terapêuticos, tecnológicos e gerenciais específicos, que viabilizem o acesso aos serviços de urgência/emergência hospitalar com garantia de acolhimento, da primeira atenção qualificada e resolutiva, da estabilização das funções vitais e da referência adequada dos pacientes graves.

Neste panorama, este artigo apresenta uma contribuição ao debate sobre a regionalização da assistência hospitalar no país, ao avaliar a rede de atenção às urgência/emergências sob a ótica de unidades de referência macro e microrregional em Pernambuco. A relevância e a oportunidade dessa avaliação ganha destaque diante do persistente e histórico imperativo de reestruturação desta rede, que está envolta na implantação dos referidos programas de qualificação das urgências/emergências regionalizadas.

\section{Métodos}

\section{Desenho do estudo}

Estudo avaliativo, do tipo normativo, definindose o grau de implantação nos serviços de urgências/emergências, comparando as características de estrutura e processo de trabalho observadas às normatizações preconizadas para o adequado funcionamento do setor. Identificaram-se ainda os fatores limitantes da assistência apontados pelos profissionais entrevistados, na intenção de subsidiar a estruturação da rede assistencial nesta área específica.

\section{Abrangência do estudo}

O território político-administrativo de Pernambuco encontra-se dividido em 11 Gerências Regionais de Saúde (GERES), que correspondem ao respectivo número de microrregiões (referência para média complexidade assistencial), estando estas, por sua vez, agrupadas conformando três macrorregiões referências para alta complexidade: Região Metropolitana, Agreste e Sertão. A rede de serviços é composta por 2.989 unidades ambulatoriais e 329 hospitais/unidades mistas, com 19.100 leitos; destacando 90 hospitais de pequeno porte, localizados em municípios com menos de 30 mil habitantes, oito hospitais microrregionais de médio porte situados nas sedes das microrregiões e sete hospitais macrorregionais de grande porte situados em Recife, Caruaru e Petrolina 10.

Para esta pesquisa foram selecionados, de forma intencional, dez hospitais, de modo a representar as unidades de referência macro e microrregional distribuídas no território estadual. Nas unidades de referência macrorregional, foram abordados os hospitais distribuídos nos três municípios sede de macrorregião, sendo estudadas as três maiores unidades de referência hospitalar em Recife; além dos dois hospitais de referência nos municípios sede de macrorregião no interior do estado: Caruaru e Petrolina. Além desses, optou-se por cinco entre os oito hospitais de referência microrregional, distribuídos em municípios sede das GERES correspondentes às microrregiões na zona da mata norte, zona da mata sul, agreste e sertão, onde devido à extensão territorial do sertão pernambucano, foram selecionados dois hospitais nesta mesorregião (Tabela 1). 
Tabela 1

Caracterização dos hospitais de referência macrorregional e microrregional avaliados por município-sede, Gerência Regional de Saúde (GERES).

\begin{tabular}{|c|c|c|c|c|c|c|}
\hline Categoria & Hospital & Município-sede & Mesorregião & GERES & $\begin{array}{c}\text { Leitos } \\
\text { hospitalares (n) }\end{array}$ & $\begin{array}{l}\text { Leitos de } \\
\text { UTI (n) }\end{array}$ \\
\hline \multirow{5}{*}{$\begin{array}{l}\text { Referência } \\
\text { macrorregional }\end{array}$} & Hospital da Restauração & Recife & Metropolitana & I & 538 & 43 \\
\hline & Hospital Getúlio Vargas & Recife & Metropolitana & I & 323 & 18 \\
\hline & Hospital Agamenon Magalhães & Recife & Metropolitana & I & 407 & 85 \\
\hline & Hospital Regional do Agreste & Caruaru & Agreste & IV & 163 & 10 \\
\hline & Hospital Dom Malan & Petrolina & Sertão & VIII & 162 & 16 \\
\hline \multirow{5}{*}{$\begin{array}{l}\text { Referência } \\
\text { microrregional }\end{array}$} & Hospital Regional de Limoeiro & Limoeiro & Mata Norte & II & 66 & - \\
\hline & Hospital Regional de Palmares & Palmares & Mata Sul & III & 105 & - \\
\hline & Hospital Regional Dom Moura & Garanhuns & Agreste & V & 110 & 3 \\
\hline & Hospital Regional Rui de Barros Correia & Arcoverde & Sertão & VI & 155 & 6 \\
\hline & Hospital Professor Agamenon Magalhães & Serra Talhada & Sertão & $X I$ & 91 & - \\
\hline
\end{tabular}

Fonte: Cadastro Nacional de Estabelecimentos de Saúde (http://cnes.datasus.gov.br/Lista_Tot_Es_Municipio.asp?Estado=26\&NomeEstado=PERNAMBUCO, acessado em 13/Out/2009).

\section{Instrumento e coleta dos dados}

Elaborou-se um instrumento considerando os critérios de avaliação do questionário validado pelo Programa Nacional de Avaliação de Serviços de Saúde (PNASS) 11. O questionário apresentou perguntas estruturadas com respostas dicotômicas do tipo "sim" ou "não", abrangendo critérios de avaliação relativos a duas dimensões: estrutura (sub-dimensões: estrutura física e organizacional, recursos materiais e humanos) e processo (sub-dimensões: atividades assistenciais de rotina e de referência e contra-referência). Na abordagem aos fatores limitantes para a assistência, sob a ótica dos entrevistados, adicionou-se ao instrumento uma questão estruturada relativa a este aspecto.

A coleta de dados ocorreu entre dezembro de 2006 e janeiro de 2007, contando com a participação de entrevistadores previamente treinados quanto ao consenso relativo aos objetivos do questionário e à clareza na exposição das questões. As entrevistas foram realizadas seguindo um cronograma que contemplou quatro plantões em cada hospital (dois em dias úteis e dois durante o final de semana). Neste cenário, em cada plantão foram entrevistados o médico e o enfermeiro responsáveis pelo setor de urgência/emergência. Nos hospitais macrorregionais onde o setor de urgência/emergência é dividido por especialidades (adulta e pediátrica), foram realizadas entrevistas com os respectivos profissionais responsáveis pelo setor especializado. Totalizaram-se 108 entrevistas, das quais 57 (52,8\%) com médicos e $51(47,2 \%)$ com enfermeiros, destacando-se que quatro profissionais se recusaram a participar da entrevista.

\section{Análise dos dados}

Seguindo a classificação do PNASS, atribuiu-se a cada critério de avaliação uma pontuação de acordo com o grau de importância dos mesmos para a assistência à urgência/emergência. Aos critérios classificados como indispensável (I) conferiram-se 3 pontos, como necessário (N) 2 pontos e aos com característica recomendável (R) 1 ponto, conforme será explicitado nas Tabelas 2 e 3 na seção dos Resultados. Atribuiu-se ainda peso 4 para os critérios relativos à dimensão estrutura e peso 6 para aqueles relacionados à dimensão processo. A pontuação final do hospital foi dividida pelo número de entrevistas realizadas a fim de se obter a pontuação média do hospital permitindo a comparabilidade entre os hospitais, uma vez que o número de entrevistas variou entre estes. Dessa forma, tem-se que: $\mathrm{PMH}=$ $(\mathrm{PE} \times 4)+(\mathrm{PP} \times 6) / \mathrm{N}$, onde: $\mathrm{PMH}=$ pontuação média do hospital; $\mathrm{PE}=$ pontuação obtida na dimensão estrutura; $\mathrm{PP}=$ pontuação obtida na dimensão processo e $\mathrm{N}$ = número de entrevistas realizadas no hospital.

O grau de implantação (GI) foi definido como o percentual da pontuação média do hospital em relação à pontuação máxima (caracterizada pela obtenção de respostas positivas a todos os critérios de avaliação). Conforme o percentual obtido, os hospitais foram classificados como: satisfatório (se atingiu de $80 \%$ a $100 \%$ da pontuação máxima), aceitável (70\% a 79\% da pontua- 
ção máxima) e deficiente (percentual inferior a $70 \%$ da pontuação máxima). Esta classificação apresentou, intencionalmente, rígidos pontos de corte, devido à natureza do objeto de estudo que corresponde a um serviço de saúde essencial à manutenção da vida.

Avaliou-se o grau de implantação geral (abrangendo todos os hospitais avaliados), por categoria (referência macrorregional e referência microrregional) e estratificado por dimensões - estrutura e processo, obtendo-se, respectivamente, grau de implantação geral, grau de implantação por dimensão, grau de implantação macrorregional e grau de implantação microrregional.

Os dados foram processados no programa SPSS 13.0 for Windows (SPSS Inc., Chicago, Estados Unidos), sendo as informações expressas sob a forma de proporção e apresentadas em tabelas de distribuição simples de freqüência.

O estudo foi aprovado pelo Comitê de Ética em Pesquisa do Centro de Pesquisas Aggeu Magalhães, Fundação Oswaldo Cruz.

\section{Resultados}

Diante dos critérios adotados, esta avaliação demonstrou que nenhum dos hospitais estudados alcançou grau de implantação satisfatório (GI = $80 \%$ a $100 \%$ ) e que seis entre as dez unidades avaliadas foram classificadas como deficiente (GI = inferior a 70\%). Entre os demais hospitais que se enquadraram como aceitável (GI $=70 \%$ a $79 \%$ ), todos são unidades localizadas em municípios de referência macrorregional.

Analisando as dimensões definidas, os hospitais de referência macrorregional apresentaram grau de implantação deficiente $(\mathrm{GI}=67,5 \%)$ para a dimensão estrutura, embora as duas unidades localizadas no interior do estado (Caruaru e Petrolina) tenham se enquadrado como aceitável (GI $=70,6 \% ; 71,5 \%$ ), acompanhando a classificação de três dos cinco hospitais microrregionais nessa dimensão. De modo inverso, nos aspectos relativos ao processo de trabalho, o desempenho das unidades macrorregionais foi suficiente para alcance do grau de implantação aceitável (GI = $74 \%$ ), diferenciando-se daqueles de referência microrregional que, com grau de implantação de $59,7 \%$, foram classificados como deficiente (Tabela 4).

Os critérios que determinaram os referidos graus de implantação demonstraram que na dimensão estrutura, apesar da sub-dimensão "estrutura organizacional" ter atingido grau de implantação satisfatório (GI = 81\%), destacou-se que, se por um lado $99 \%$ dos entrevistados indi- caram a presença de prontuário de atendimento individual, apenas 36\% dos hospitais estudados apresentam normas de funcionamento e atendimento às urgências/emergências.

Nas sub-dimensões "recursos materiais e recursos humanos", consequentemente graus de implantação aceitável (GI = 68\%) e deficiente ( $\mathrm{GI}=39 \%$ ), observou-se que somente $27 \%$ dos entrevistados apontaram que as unidades dispõem de equipamentos/materiais suficientes e em funcionamento; bem como a conformação de uma equipe multiprofissional em tempo integral foi indicada por apenas $9 \%$ dos entrevistados. Observou-se ainda que se nos hospitais de referência macrorregional, 65\% dos entrevistados apontaram que os profissionais têm capacitação específica para assistência aos casos de urgência/emergência, nos hospitais microrregionais este percentual foi de apenas $37 \%$ (Tabela 2).

A abordagem da dimensão processo demonstrou que na sub-dimensão "atividades de rotina" ( $\mathrm{GI}=66 \%$ ), 90\% dos entrevistados indicaram que os serviços oferecem exames laboratoriais, 99\% mencionaram que estes cumprem o apoio medicamentoso em tempo integral, além de a relação profissional-acompanhante para informação do estado geral do paciente ser estabelecida, conforme $93 \%$ dos entrevistados. Contudo, a minoria dos médicos e enfermeiros abordados (32\%) afirmou que os serviços assistem os casos de emergência (situações com risco de vida) e apenas $11 \%$ apontaram para a oferta de exames por imagem em todos os plantões, sejam estes próprios (radiografia, endoscopia, eletrocardiografia) ou conveniados (ressonância magnética, tomografia e ultrassonografia) (Tabela 3).

Ressalta-se ainda a realização de triagem dos pacientes sem risco de vida que foi indicada por $59 \%$ dos entrevistados nos hospitais de referência macrorregional, mas por apenas $20 \%$ dos entrevistados nos hospitais microrregionais. Inversamente, a permanência do paciente nos serviços de urgência/emergência em até 24 horas foi apontada por $55 \%$ dos entrevistados nos hospitais microrregionais e por apenas $25 \%$ daqueles abordados nos hospitais de referência macrorregional. Ademais, embora o encaminhamento para UTI no próprio hospital após procedimentos clínicos esteja incorporado à rotina do serviço, segundo $80 \%$ dos entrevistados nas unidades de referência macrorregional, apenas $38 \%$ dos entrevistados nas unidades microrregionais responderam positivamente a essa questão (Tabela 3).

Nesse cenário, os entrevistados em ambas as categorias hospitalares estudadas identificaram como principais entraves para a prestação da assistência à urgência/emergência a demanda 
Tabela 2

Distribuição percentual dos critérios relativos à estrutura necessária à atenção às urgências e emergências, segundo os hospitais de referência macrorregional e microrregional de Pernambuco, Brasil.

\begin{tabular}{|c|c|c|c|c|}
\hline Sub-dimensão & Critérios relativos à estrutura & $\begin{array}{c}\text { Referência } \\
\text { macrorregional } \\
(n=69)\end{array}$ & $\begin{array}{c}\text { Referência } \\
\text { microrregional } \\
(n=39)\end{array}$ & $\begin{array}{c}\text { Total } \\
(n=108)\end{array}$ \\
\hline \multirow[t]{8}{*}{ Estrutura física } & Local exclusivo/acesso independente ao atendimento imediato (I) & 88 & 82 & 86 \\
\hline & Dispositivos para portadores de limitações físicas (I) & 48 & 44 & 46 \\
\hline & Espaços diferenciados (consultas/procedimentos/observação) (I) & 57 & 82 & 66 \\
\hline & Centro cirúrgico (I) & 81 & 97 & 87 \\
\hline & Centro obstétrico (R) & 41 & 95 & 60 \\
\hline & Enfermarias para acomodação após procedimentos cirúrgicos (N) & 90 & 92 & 91 \\
\hline & $\begin{array}{l}\text { Locais com condição de lavagem simples e anti-sepsia das mãos, } \\
\text { ventilação e iluminação adequadas (I) }\end{array}$ & 68 & 90 & 76 \\
\hline & Grau de implantação/Dimensão & 69 & 80 & 73 \\
\hline \multirow[t]{6}{*}{$\begin{array}{l}\text { Estrutura } \\
\text { organizacional }\end{array}$} & $\begin{array}{l}\text { Normas de rotina de funcionamento e atendimento da assistência às } \\
\text { urgências/emergências (R) }\end{array}$ & 43 & 35 & 36 \\
\hline & $\begin{array}{l}\text { Normas, rotinas e procedimentos em relação ao controle da infecção } \\
\text { hospitalar (R) }\end{array}$ & 61 & 45 & 52 \\
\hline & Prontuário para cada paciente (N) & 99 & 100 & 99 \\
\hline & Ficha própria para descrição de ato cirúrgico $(\mathrm{N})$ & 90 & 97 & 92 \\
\hline & Banco de sangue próprio ou previamente pactuado (R) & 99 & 98 & 98 \\
\hline & Grau de implantação/Dimensão & 83 & 78 & 81 \\
\hline \multirow[t]{4}{*}{ Recursos materiais } & Equipamentos/materiais em funcionamento e quantidade suficiente (I) & 30 & 30 & 27 \\
\hline & Medicamentos básicos em quantidade suficiente (I) & 88 & 100 & 93 \\
\hline & Ambulância e/ou UTI móvel & 94 & 90 & 93 \\
\hline & Grau de implantação/Dimensão & 68 & 68 & 68 \\
\hline \multirow[t]{6}{*}{ Recursos humanos } & Equipe multiprofissional e especializada em tempo integral (N) & 7 & 13 & 9 \\
\hline & Profissionais com capacitação específica (R) & 65 & 37 & 51 \\
\hline & $\begin{array}{l}\text { Equipe dimensionada em quantidade e qualidade para prestar o } \\
\text { atendimento com o grau de risco e necessidade }(N)\end{array}$ & 26 & 17 & 20 \\
\hline & Enfermeiro intitulado responsável pelo corpo de enfermagem (R) & 99 & 85 & 91 \\
\hline & Médico especialista chefe de plantão (R) & 99 & 55 & 74 \\
\hline & Grau de implantação/Dimensão & 47 & 26 & 39 \\
\hline
\end{tabular}

I: indispensável; N: necessário; R: recomendável.

ser maior que a oferta (95\%), o hospital funcionar como porta de entrada do sistema de saúde (86\%) e os profissionais não receberem capacitação específica (69\%) (Tabela 5).

\section{Discussão}

A larga dimensão geográfica brasileira, a dinâmica de evolução de modelos assistenciais (organização e gestão) e de financiamento, a redefinição do perfil demográfico e epidemiológico, do poder corporativo-institucional, perpassando ainda pelos valores e representações sociais da comunidade em relação ao hospital, refletido no modelo hospitalocêntrico, agregam complexidade e desafios aos processos de gestão e assistência em redes de saúde integradas e regionalizadas 12,13 . Esse quadro pressiona, sobretudo, a qualificação de serviços de alto custo e tecnologia, evidenciando aqueles essenciais à manutenção da vida, como as unidades de urgência/emergência hospitalar.

Nessa conjuntura, o presente estudo recorreu a uma abordagem normativa, como ferramenta factível e de simples reprodução para avaliação de ambientes hospitalares de urgência/emergência. A peculiaridade deste tipo de avaliação 
Tabela 3

Distribuição percentual dos critérios relativos ao processo de trabalho necessário à atenção às urgências/emergências, segundo os hospitais de referência macrorregional e microrregional de Pernambuco, Brasil.

\begin{tabular}{|c|c|c|c|c|}
\hline Sub-dimensão & Critérios relativos ao processo & $\begin{array}{c}\text { Referência } \\
\text { macrorregional } \\
(n=69)\end{array}$ & $\begin{array}{c}\text { Referência } \\
\text { microrregional } \\
(n=39)\end{array}$ & $\begin{array}{l}\text { Total } \\
(\mathrm{n}=108)\end{array}$ \\
\hline \multirow{20}{*}{$\begin{array}{l}\text { Atividades de } \\
\text { rotina }\end{array}$} & Atendimento de urgência em tempo integral (I) & 78 & 82 & 76 \\
\hline & Atendimento de emergência em tempo integral (I) & 36 & 38 & 32 \\
\hline & Triagem para os casos sem risco de vida (N) & 59 & 20 & 46 \\
\hline & $\begin{array}{l}\text { Exames por imagem em tempo integral (ultrassonografia, endoscopia e } \\
\text { ressonância magnética) (I) }\end{array}$ & 16 & 8 & 11 \\
\hline & Exames laboratoriais em tempo integral (I) & 93 & 85 & 90 \\
\hline & Apoio medicamentoso em tempo integral $(\mathrm{N})$ & 99 & 100 & 99 \\
\hline & $\begin{array}{l}\text { Rotina de acompanhamento/evolução dos pacientes (médico e } \\
\text { enfermeiro) (N) }\end{array}$ & 91 & 90 & 89 \\
\hline & Acesso imediato ao centro cirúrgico em casos de emergência (I) & 75 & 87 & 77 \\
\hline & $\begin{array}{l}\text { Encaminhamento para sala de recuperação após procedimentos } \\
\text { cirúrgicos }(\mathrm{N})\end{array}$ & 88 & 78 & 82 \\
\hline & Higiene pessoal do paciente $(\mathrm{R})$ & 99 & 93 & 96 \\
\hline & Atendimento social ao paciente/acompanhante (R) & 68 & 88 & 93 \\
\hline & Procedimentos rotineiros de controle de infecção hospitalar (N) & 64 & 72 & 66 \\
\hline & Permanência do paciente no hospital em até 24 horas (N) & 25 & 55 & 43 \\
\hline & $\begin{array}{l}\text { Protocolos clínicos de urgência e emergência na prática diária dos } \\
\text { profissionais (R) }\end{array}$ & 65 & 48 & 56 \\
\hline & $\begin{array}{l}\text { Organização de grupos de trabalho para discussão de casos clínicos/ } \\
\text { cirúrgicos e para integração institucional (R) }\end{array}$ & 22 & 27 & 20 \\
\hline & Fornecimento de refeição com rotina de suporte nutricional (R) & 97 & 85 & 91 \\
\hline & Informação aos acompanhantes do estado geral do paciente (R) & 99 & 88 & 93 \\
\hline & $\begin{array}{l}\text { Encaminhamento para UTI no próprio hospital após procedimentos } \\
\text { clínicos (I) }\end{array}$ & 80 & 38 & 65 \\
\hline & $\begin{array}{l}\text { Encaminhamento para UTI no próprio hospital após procedimentos } \\
\text { cirúrgicos (I) }\end{array}$ & 91 & 67 & 78 \\
\hline & Grau de implantação/Dimensão & 69 & 59 & 66 \\
\hline \multirow{7}{*}{$\begin{array}{l}\text { Referência e } \\
\text { contra-referência } \\
\text { assistencial }\end{array}$} & $\begin{array}{l}\text { Retaguarda de maior complexidade previamente pactuada, com fluxos } \\
\text { e mecanismos de transferência claros }(\mathrm{N})\end{array}$ & 80 & 78 & 75 \\
\hline & Fluxos para elucidação diagnóstica previamente pactuada (N) & 96 & 62 & 77 \\
\hline & Unidade mediada por central de regulação (N) & 99 & 95 & 97 \\
\hline & Comunicação efetiva entre médicos e centrais de regulação (R) & 99 & 93 & 95 \\
\hline & Referência para unidade de maior complexidade (R) & 94 & 63 & 76 \\
\hline & Contra-referência para a atenção básica $(R)$ & 71 & 42 & 51 \\
\hline & Grau de implantação/Dimensão & 90 & 62 & 80 \\
\hline
\end{tabular}

I: indispensável; N: necessário; R: recomendável. 
Tabela 4

Grau de implantação da atenção à urgência/emergência segundo os hospitais de referência macrorregional e microrregional de Pernambuco, Brasil.

\begin{tabular}{|c|c|c|c|}
\hline \multirow[t]{3}{*}{ Categorias/Hospitais } & \multicolumn{3}{|c|}{ Grau de implantação (\%) } \\
\hline & \multicolumn{2}{|c|}{ Dimensão } & \multirow[t]{2}{*}{ Geral } \\
\hline & Estrutura & Processo & \\
\hline \multicolumn{4}{|l|}{ Referência macrorregional } \\
\hline Hospital da Restauração & 67,5 & 78,5 & 74,6 \\
\hline Hospital Getúlio Vargas & 66,9 & 77,7 & 73,9 \\
\hline Hospital Agamenon Magalhães & 63,3 & 62,9 & 63,0 \\
\hline Hospital Regional do Agreste & 70,6 & 75,5 & 73,8 \\
\hline Hospital Dom Malan & 71,5 & 76,6 & 74,8 \\
\hline Grau de implantação macrorregional & 67,5 & 74,0 & 71,7 \\
\hline \multicolumn{4}{|l|}{ Referência microrregional } \\
\hline Hospital Regional de Limoeiro & 73,1 & 50,8 & 58,8 \\
\hline Hospital Regional de Palmares & 71,9 & 62,8 & 66,0 \\
\hline Hospital Regional Dom Moura & 74,1 & 58,1 & 63,8 \\
\hline Hospital Regional Rui de Barros Correia & 57,2 & 65,1 & 62,3 \\
\hline Hospital Professor Agamenon Magalhães & 64,6 & 40,0 & 63,1 \\
\hline Grau de implantação microrregional & 68,3 & 59,7 & 62,8 \\
\hline Grau de implantação geral & 67,8 & 68,8 & 68,5 \\
\hline
\end{tabular}

Satisfatório (80\% a 100\%)

Aceitável (70\% a 79\%)

Deficiente $(<70 \%)$

Tabela 5

Distribuição percentual dos entraves apontados pelos profissionais na atenção às urgências/emergências de Pernambuco, Brasil.

\begin{tabular}{|c|c|c|c|}
\hline Entraves apontados & $\begin{array}{c}\text { Referência } \\
\text { macrorregional } \\
(n=69)\end{array}$ & $\begin{array}{c}\text { Referência } \\
\text { microrregional } \\
(n=39)\end{array}$ & $\begin{array}{c}\text { Total } \\
(n=108)\end{array}$ \\
\hline Gestão hospitalar vinculada à gestão estadual & 4 & 13 & 7 \\
\hline Orçamento hospitalar insuficiente & 38 & 44 & 40 \\
\hline $\begin{array}{l}\text { Capacitação profissional específica para urgência e emergência } \\
\text { insuficiente }\end{array}$ & 64 & 80 & 69 \\
\hline Demanda superior à capacidade de oferta de serviços & 100 & 87 & 95 \\
\hline Atendimento à demanda de atenção primária e secundária & 84 & 90 & 86 \\
\hline Vínculo profissional precário & 28 & 44 & 33 \\
\hline
\end{tabular}

abrange ainda a possibilidade de atribuição de pesos às dimensões, sendo ponderado, nesta avaliação, que para o alcance da assistência efetiva aos casos de urgência/emergência, a implantação da dimensão processo apresenta maior peso do que a plena implantação da estrutura. O posicionamento foi voluntário para evidenciar o desenvolvimento do processo de trabalho e em decorrência o redirecionamento das ações prioritárias para aumentar o grau de implantação da Rede Estadual de Urgência/Emergência Hospitalar.

Com esta abordagem, os resultados observados corroboraram outros estudos que abordaram 
o tema em questão 14,15,16,17. Demonstraram-se fortes limitações relativas aos cuidados às urgências/emergências; sobretudo nos hospitais classificados como deficiente, representados por unidades microrregionais e por um serviço de urgência/emergência, referência macrorregional e unidade de ensino.

Os distintos graus de implantação entre as unidades hospitalares avaliadas evidenciaramse, mormente, na abordagem estratificada por dimensão (estrutura e processo) que descortinou convergências e divergências significativas entre as categorias hospitalares analisadas (referência microrregional e macrorregional).

Predominantemente, os hospitais de referência microrregional apresentaram desempenho mais favorável para os critérios relativos à dimensão estrutura, enquanto que nos hospitais de referência macrorregional o processo de trabalho apresentou melhor grau de implantação. Em consonância, essa última categoria apresentou desempenho geral mais favorável. Destacase nesse aspecto que, além de os melhores graus de implantação do processo de trabalho possivelmente terem atenuado as fragilidades estruturais, os hospitais macrorregionais são de grande porte, o que de acordo com Mendes 13 significa que apresentam maior economia de escala, e escopo e consequentemente melhores resultados, devido ao volume e à especialização de procedimentos. Da mesma forma, a taxa de ocupação hospitalar das referidas unidades é superior aos hospitais de médio porte, como a categoria de referência microrregional.

Influenciando fortemente a implantação dos serviços de urgência/emergência nos hospitais sob análise, a deficiente conformação de equipes multiprofissionais e especializada em regime de plantão de 24 horas destacou-se como nó crítico a ser superado. As questões que circundam a relação entre os recursos humanos e a qualidade dos serviços de saúde e, sobretudo os de assistência às urgências/emergências, são complexas e eventualmente complementares. Ainda são uma constante os ambientes hospitalares com o desafio de ultrapassar os limites da conformação da equipe multiprofissional em busca da efetiva prática interdisciplinar 18 , sendo persistente a defasagem salarial, com fragilidade do vínculo profissional com a instituição pública e com a função que exerce, frente à superlotação das unidades 17. Em decorrência há insatisfação profissional gerada pelo arranjo de questões que envolvem a sua atuação e o excesso de trabalho, resultando em desgaste físico-emocional dos profissionais de saúde 19 .

Contribuindo para o delineamento deste quadro, estudo realizado por Deslandes 20 chama atenção para a desestruturação técnica e operativa das equipes de plantão e despreparo no atendimento dos casos nos serviços de emergência. Tais situações direcionam para a necessidade de intervenções governamentais estruturadas na direção da suficiência de profissionais, de forma a subsidiar o desenvolvimento de políticas de planos e carreiras, contemplando o enfrentamento de fragilidades de vínculos profissionais e oportunidades de qualificação profissional, com destaque ao âmbito microrregional. Sob esse último aspecto, iniciativas de educação permanente em saúde deverão ser fortalecidas na direção da interiorização, superando as desigualdades regionais, as necessidades de formação e desenvolvimento para o trabalho em saúde.

Paralelamente ao comprometimento de recursos humanos observou-se nesse estudo a insuficiência de materiais e equipamentos essenciais, além do comprometimento da oferta de exames por imagem. Por outro lado, a estruturação dos serviços de transporte (ambulância e UTI móvel) no conjunto dos hospitais avaliados, confrontou-se com a inexistente ou insuficiente agregação de tecnologia como a UTI, sobretudo nas unidades microrregionais.

Em consonância com a grande inadequação na oferta de leitos de UTI no Brasil, caracterizada pela concentração regional e comprometimento funcional desses equipamentos 21 , os hospitais no interior do estado agregam 35 leitos de UTI, distribuídos em somente quatro hospitais 22 . Considerando que duas dessas unidades integram os hospitais de referência macrorregional avaliados neste estudo, sugere-se que a deficiência estrutural nos hospitais microrregionais contribua para a manutenção da rotina de transferência de emergências clínicas e cirúrgicas graves aos hospitais macrorregionais. Cabe destacar, entretanto, que na extensão territorial do estado - 98.312km² - (Instituto Brasileiro de Geografia e Estatística. Estados@. http://www.ibge.gov.br/ estadosat/perfil.php?sigla=pe, acessado em 20/ Nov/2009), a trajetória por rodovias públicas em condições de incipiente adequação estrutural poderá ser pouco profícua diante de situações graves com risco de morte.

Tais deficiências ainda corroboram uma prática assistencial centrada nos casos de menor gravidade, com a carência de normas de atendimento específicas ao setor, a incipiente rotina de triagem dos pacientes e permanência do usuário em período superior a 24 horas nos serviços de urgência/emergência, como observado de forma relevante no presente estudo. Esse quadro descaracterizou a assistência aos casos de emergência, conduzindo ainda à superlotação das unidades, sendo este aspecto ressaltado pelos entrevista- 
dos como importante entrave. Assim, a despeito da subjetividade agregada à triagem de pacientes em situações de emergência, faz-se necessário o fortalecimento de estratégias de classificação dos usuários por grau de risco de morte 14; assim como da instituição de protocolos clínicos, que está relacionada à melhoria dos padrões assistenciais e à redução da superlotação nos serviços de urgência/emergência hospitalar 23.

Diante dos resultados obtidos neste estudo, a implantação de redes assistenciais, em que o espaço regional seja o cenário para gestão e assistência de média e alta complexidade hospitalar, demonstrou-se ainda incipiente. Por outro lado, a evidência das fragilidades assistenciais pode subsidiar o planejamento loco-regional de ações com distintos níveis de complexidade, no tocante à prioridade, viabilidade financeira e aos prazos de execução.

Neste sentido, é importante ressaltar que com a constatação dessa problemática situação que se instala historicamente no SUS, emergemse os desafios de reestruturação e recentes tendências de gestão dos serviços hospitalares, haja vista o inegável papel que este setor representa. A transição de uma regionalização genuinamente político-administrativa à outra que incorpore o caráter funcional-assistencial urge como prioridade das três instâncias de governo.

Em Pernambuco, para além da implementação das normatizações federais, foram instituídas como metas do Plano Plurianual (2008/2011) a reestruturação dos hospitais microrregionais, com fortalecimento da Rede Pré-hospitalar de Baixa Complexidade, além da ampliação da Rede Hospitalar de Urgência/Emergência, redistribuindo a demanda concentrada nas grandes emergências e cumprindo os objetivos da regionalização. Ademais, previu a instalação de Fundações Estatais de Direito Privado, promulgando a Lei Estadual no $n^{\circ} 13.53724$ com o objetivo de instituir a Fundação Estadual de Assistência Hospitalar Josué de Castro para administração pública indireta da assistência hospitalar.

A despeito das referências de inconstitucionalidade desse modelo de administração, desde 2008 três hospitais macrorregionais e uma unidade microrregional, avaliadas no presente estudo, estão sob administração indireta. Deve-se, de fato ponderar as lacunas e benefícios da proposta em questão, pois esta representa o compromisso com a eficiência e efetividade por meio da administração dos serviços públicos. Prevê o estabelecimento de planos operativos, com vistas ao cumprimento de metas coerentes com o SUS, assim como aborda a fixação profissional em municípios interioranos sede dos hospitais microrregionais. Por outro lado, há riscos agregados à flexibilidade das licitações públicas, ilimitação na remuneração profissional, bem como à incompatibilidade entre regime de direito privado e a prestação de serviços públicos de assistência à saúde, atividade típica do poder público 25 .

Não obstante os processos em evolução no SUS, os princípios constitucionais devem ser respeitados e perseguidos. Da mesma forma, os diversos aspectos comprometedores da qualidade da assistência hospitalar, como os relacionados aos recursos humanos e à organização de Redes de Atenção Integradas merecem não apenas ser objeto de avaliação, mas de efetiva intervenção para que estas questões não permaneçam intactas, como ainda foi sensivelmente observado no contexto de Pernambuco. 


\section{Resumo}

Este artigo avalia o grau de implantação de dez serviços de urgência/emergência hospitalar, que compõem a rede de referência macro e microrregional em Pernambuco, Nordeste brasileiro. Aborda critérios relativos à estrutura (física-organizacional, recursos materiais e humanos) e ao processo (atividades de rotina e de referência/contra-referência); classificando os hospitais como satisfatório, aceitável e deficiente. Aponta que a maioria dos hospitais se classifica como deficiente, estando entre estes todas as unidades de referência microrregionais e uma macrorregional. Estas últimas apresentam melhor desempenho quanto ao processo de trabalho; e a dimensão estrutura apresenta-se mais bem implantada nos hospitais microrregionais. Os resultados evidenciam como prioridade a qualificação dos hospitais avaliados, mediante o fortalecimento de políticas descentralizadas de recursos humanos e tecnológicos, direcionados à melhoria das práticas de trabalho consensuais aos contextos regionais do estado.

Serviço Hospitalar de Emergência; Avaliação em Saúde; Regionalização

\section{Referências}

1. Arretche TS. Políticas sociais no Brasil: descentralização em um estado federativo. Rev Bras Ciênc Soc 1999; 14:111-41.

2. Heimann LS, Cortizo CT, Castro IEN, Kayano J, Rocha JL, Nascimento PR, et al. Descentralização do Sistema Único de Saúde: trilhando a autonomia municipal. São Paulo: Sociedade Brasileira de Vigilância de Medicamentos; 2000.

3. Lucchese PTR. Descentralização do financiamento e gestão da assistência à saúde no Brasil: a implementação do Sistema Único de Saúde - retrospectiva 1990/1995. Planej Polít Públicas 1996; 14: 75-156.

4. Mendes EV. A organização da saúde no nível local. São Paulo: Editora Hucitec; 1998.

5. Ministério da Saúde. Portaria GM nº. 399, de 22 de fevereiro de 2006. Divulga o Pacto pela Saúde 2006, Consolidação do SUS. Dispõe sobre a Regionalização da assistência à saúde, aprofundando a descentralização com eqüidade no acesso. http:// dtr2001.saude.gov.br/sas/PORTARIAS/Port2006/ GM/GM-399.htm (acessado em 19/Jan/2008).

\section{Colaboradores}

L. S. Dubeux participou da concepção do projeto, pesquisa de campo, análise e interpretação dos dados; concepção e redação final. E. Freese contribuiu na concepção do projeto e redação final. Y. A. C. Reis colaborou na pesquisa de campo e concepção do artigo.
6. Santos NR. Desenvolvimento do SUS, rumos estratégicos e estratégias para visualização dos rumos. Ciênc Saúde Coletiva 2007; 12:429-35

7. Ministério da Saúde. Portaria GM nº. 2048, de 5 de novembro de 2002. Dispõe sobre Sistemas estaduais de urgência e emergência: regulamento técnico. http://dtr2001.saude.gov.br/sas/PORTARIAS/ Port2002/Gm/GM-2048.htm (acessado em 11/ Dez/2008).

8. Ministério da Saúde. QualiSUS. http://portal.sau de.gov.br/SAUDE/area.cfm?id_area=408 (acessado em 07/Set/2009).

9. Brasil. Portaria GM no. 2.922, 2 de dezembro de 2008. Estabelece diretrizes para organização das redes loco-regionais de atenção integral às urgências. http://dtr2001.saude.gov.br/sas/PORTARIAS/ Port2008/GM/GM-2922.htm (acessado em 24/ Set/2009). 
10. Secretaria Estadual de Saúde de Pernambuco Plano Diretor de Regionalização - PDR SUS/PE. http://www.saude.pe.gov.br/site/regionalizacao/ InstrumentosdePlanejamento/pdr_completo_ pe.pdf (acessado em 23/Jul/2008).

11. Coordenação Geral de Regulação e Avaliação, Departamento de Regulação, Avaliação e Controle de Sistemas, Secretaria de Atenção à Saúde, Ministério da Saúde. PNASS - Programa Nacional de Avaliação de Serviços de Saúde. Edição 2004/2005. Brasília: Ministério da Saúde; 2004.

12. Brasil. Portaria GM n ${ }^{\circ}$. 2468, de 13 de outubro de 2006. Constitui Grupo de Trabalho com o objetivo de apresentar proposta de regulamentação para a Política de Qualidade dos Serviços de Saúde Hospitalar no âmbito do Sistema Único de Saúde - SUS. http://dtr2001.saude.gov.br/sas/PORTA RIAS/Port2006/GM/GM-2468.htm (acessado em $11 / \operatorname{Jan} / 2009$ ).

13. Mendes EA. Revisão bibliográfica sobre redes de atenção à saúde. Belo Horizonte: Superintendência de Atenção à Saúde, Subsecretaria de Políticas e Ações em Saúde, Secretaria de Estado de Saúde de Minas Gerais; 2007.

14. Bittencourt RJ, Hortale VA. A qualidade nos serviços de emergência de hospitais públicos e algumas considerações sobre a conjuntura recente no município do Rio de Janeiro. Ciênc Saúde Coletiva 2007; 12:929-34.

15. Mendes ACG, Araújo Júnior JLCA, Furtado BMAS Duarte PO, Santiago RF, Costa TR. Avaliação da satisfação dos usuários com a qualidade do atendimento nas grandes emergências do Recife, Pernambuco, Brasil. Rev Bras Saúde Matern Infant 2009; 9:157-65.

16. Gusmão-Filho FAR. Análise de implantação da Política de Qualificação da Atenção à Saúde do Sistema Único de Saúde - Política QualiSUS - em três hospitais do município do Recife [Tese de Doutorado]. Recife: Centro de Pesquisas Aggeu Magalhães, Fundação Oswaldo Cruz; 2009.
17. O’Dwyer G, Matta IEA, Pepe VLE. Avaliação dos serviços hospitalares de emergência do estado do Rio de Janeiro. Ciênc Saúde Coletiva 2008; 13: 1637-48.

18. Vecina Neto G, Malik AM. Tendências na assistência hospitalar. Ciênc Saúde Coletiva 2007; 12 : 825-39.

19. Feliciano KVO, Kovacs MH, Sarinho SW. Sentimentos de profissionais dos serviços de pronto-socorro pediátrico: reflexões sobre o burnout. Rev Bras Saúde Matern Infant 2005; 5:319-28.

20. Deslandes SF. Frágeis Deuses: profissionais da emergência entre danos da violência e recriação da vida. Rio de Janeiro: Editora Fiocruz; 2002.

21. Castro MSM, Travassos C, Carvalho MS. Efeito da oferta de serviços de saúde no uso de internações hospitalares no Brasil. Rev Saúde Pública 2005; 39:277-84.

22. Ministério da Saúde. Distribuição percentual de leitos hospitalares de Unidade de Terapia Intensiva por Regionais de Saúde em Pernambuco. Brasília: Ministério da Saúde; 2009.

23. Bittencourt RJ, Hortale VA. Intervenções para solucionar a superlotação nos serviços de saúde de emergência hospitalar: uma revisão sistemática. Cad Saúde Pública 2009; 25:1439-54.

24. Assembléia Legislativa de Pernambuco. Lei no. 13.537, de 12 de setembro de 2008. Autoriza o Poder executivo a instituir "Fundação Estadual de Assistência Hospitalar Josué de Castro", e dá outras providências. http://www.upe.br/down/impren sa/lei13537.pdf (acessado em 11/Dez/2009).

25. Weichert MA. Fundação estatal no serviço público de saúde: inconsistências e inconstitucionalidades. Rev Direito Sanit 2009; 10:81-9.

Recebido em 15/Dez/2009

Versão final reapresentada em 17/Abr/2010

Aprovado em 03/Mai/2010 